\title{
FINE SELMER GROUP OF HIDA DEFORMATIONS OVER NON-COMMUTATIVE $p$-ADIC LIE EXTENSIONS*
}

\author{
SOMNATH $\mathrm{JHA}^{\dagger}$
}

\begin{abstract}
We study the Selmer group and the fine Selmer group of $p$-adic Galois representations defined over a non-commutative $p$-adic Lie extension and their Hida deformations. For the fine Selmer group, we generalize the pseudonullity conjecture of $[\mathrm{C}-\mathrm{S}]$ in this context and discuss its invariance in a branch of a Hida family. We relate the structure of the 'big' Selmer (resp. fine Selmer) group with the specialized individual Selmer (resp. fine Selmer) groups.
\end{abstract}

Key words. Selmer group, congruences of modular forms, Hida theory, $p$-adic Galois representation, non-commutative Iwasawa theory.

AMS subject classifications. Primary 11R23，11F33，11F80; Secondary 11G05, 14G05, $16 \mathrm{E} 40$.

Introduction. Let $E$ be an elliptic curve over a number field $K$ and let $p$ be an odd prime number. Suppose that $\mathcal{L} / K$ is a Galois extension such that the Galois group $\mathcal{G}=\operatorname{Gal}(\mathcal{L} / K)$ is a pro- $p, p$-adic Lie group with no elements of order $p$ and $\mathcal{L}$ contains the cyclotomic $\mathbb{Z}_{p}$-extension, $K_{\text {cyc }}$ of $K$. The fine Selmer group of $E$ over such $p$-adic Lie extensions $\mathcal{L}$ of $K$ is an interesting arithmetic module that is studied in Iwasawa theory. In [C-S], Coates and Sujatha formulated some conjectures about the fine Selmer group for such extensions. One of these, namely Conjecture A of [C-S] was generalized in $[\mathrm{J}-\mathrm{S}]$ for Hida families. The main aim of this article is to formulate the analogue of Conjecture B of [C-S] for the Galois representation associated to a modular form and also for a 'large' Galois representation attached to a Hida family, as enunciated by Hida theory (see [Hi]). We investigate the relationship between these two conjectures and also study, under certain hypotheses, the invariance of the $\Lambda(\mathcal{H})$-rank of the dual Selmer group and the dual fine Selmer group of the members in a branch of a Hida family where $\mathcal{H}=\operatorname{Gal}\left(\mathcal{L} / \mathrm{K}_{\text {cyc }}\right)$ and $\Lambda(\mathcal{H})$ is the usual Iwasawa algebra of $\mathcal{H}$. The article consists of four sections. Section 1 sets forth the notation and some preliminary results and in section 2, the fine Selmer group is defined and the analogues of Conjecture B are formulated. In section 3, we prove a control theorem and study the relationship between the conjecture on the 'big' fine Selmer group for a Hida family and the corresponding conjecture for the specializations. We also illustrate our main results with a numerical example. Finally in section 4, we study the $\Lambda(\mathcal{H})$-rank of the dual Selmer group in a branch of the Hida family under the additional hypothesis that cyclotomic $\mu$-invariant of the dual Selmer group vanishes.

1. Preliminaries. Throughout the article, $p$ will denote an odd prime integer and $N$ a natural number prime to $p$. Fix an embedding of an algebraic closure $\overline{\mathbb{Q}}$ of the field $\mathbb{Q}$ of rational numbers into $\mathbb{C}$ and also an embedding of $\overline{\mathbb{Q}}$ into a fixed algebraic closure $\overline{\mathbb{Q}}_{p}$ of the field $\mathbb{Q}_{p}$ of the $p$-adic numbers. Throughout, $K$ will denote an abelian number field. The cyclotomic $\mathbb{Z}_{p}$-extension of $K$ is denoted by $K_{\text {cyc }}$ with $\Gamma=\operatorname{Gal}\left(K_{\text {cyc }} / K\right) \cong \mathbb{Z}_{p}$. Let $S$ be a finite set of places of $K$ containing the primes over $N p$ and the infinite primes and let $K_{S}$ denote the maximum algebraic extension of $K$ unramified outside $S$. Let $\mathcal{L}$ be an algebraic extension of a number field $K$ such

\footnotetext{
*Received April 28, 2011; accepted for publication August 25, 2011.

†School of Mathematics, Tata Institute of Fundamental Research, Homi Bhabha Road, Mumbai 400005, India (somnath@math.tifr.res.in).
} 
that

$$
K \subset K_{\text {cyc }} \subset \mathcal{L} \subset K_{S}
$$

Put $\mathcal{G}=\operatorname{Gal}(\mathcal{L} / K)$ and assume that $\mathcal{G}$ is a pro $p, p$-adic Lie group of dimension $\geq 1$ with no $p$-torsion. We further assume that only finitely many primes of $K$ ramify in $\mathcal{L}$. Such an extension $\mathcal{L}$, is called an admissible $p$-adic Lie extension of $K$. Write $\mathcal{H}=\operatorname{Gal}\left(\mathcal{L} / K_{\text {cyc }}\right)$, so that $\mathcal{G} / \mathcal{H} \cong \Gamma \cong \mathbb{Z}_{p}$. Let $B$ be a complete, commutative, regular local ring with finite residue field. The Iwasawa algebra for a $p$-adic Lie extension $\mathcal{L}$ over the $\operatorname{ring} B$ is defined by $B[[G]]=\Lambda_{B}(\mathcal{G}):={\underset{\leftarrow}{U}}_{\lim } B[[\mathcal{G} / U]]$, where $U$ varies over an inverse system of open normal subgroups of $\mathcal{G}$. For a finite extension $O$ of $\mathbb{Z}_{p}$ and for $\mathcal{G}=\Gamma$, the Iwasawa algebra $\Lambda_{O}(\Gamma)$ is (non-canonically) isomorphic to the power series ring $O[[T]]$. The algebra $O[[T]]$ is a commutative regular local ring while $\Lambda_{O}(\mathcal{G})$ is in general, an Auslander regular domain. All modules considered over non-commutative Iwasawa algebras will be left modules. For any field $L, G_{L}$ denotes the Galois group $\operatorname{Gal}(\bar{L} / L)$. Given a field extension $L / K$ and a discrete module $M$ over the Galois group $\operatorname{Gal}(L / K)$, the Galois cohomology groups are denoted by $H^{i}(L / K, M)$. Also for a module $N$ over an Iwasawa algebra, $N^{\vee}$ denotes its Pontryagin dual.

The group $\Gamma^{\prime}$ denotes the group of diamond operators for the tower of modular curves $\left\{Y_{1}\left(p^{t}\right)\right\}_{t \geq 1}$. There are canonical isomorphisms $\chi: \Gamma \cong 1+p \mathbb{Z}_{p} \subset \mathbb{Z}_{p}^{*}$ (resp. $\kappa: \Gamma^{\prime} \cong 1+p \mathbb{Z}_{p} \subset \mathbb{Z}_{p}^{*}$ ) with $\chi$ the $p$-adic cyclotomic character. We also fix a topological generator $\gamma\left(\right.$ resp. $\left.\gamma^{\prime}\right)$ of $\Gamma$ (resp. $\Gamma^{\prime}$ ), such that $\chi(\gamma)=\kappa\left(\gamma^{\prime}\right)$. Let $\mathbb{H}_{\mathcal{F}}^{\text {ord }}$ be the quotient of the universal ordinary Hecke algebra $\mathbb{H}_{N p^{\infty}}^{\text {ord }}$, that corresponds to an ordinary $\Lambda$-adic newform $\mathcal{F}$ (see $[\mathrm{Hi}]$ ). The algebra $\mathbb{H}_{\mathcal{F}}^{\text {ord }}$ is a $\mathbb{Z}_{p}$-algebra which is a local domain and is finite flat over $\mathbb{Z}_{p}\left[\left[\Gamma^{\prime}\right]\right]$. Let

$$
\mathcal{F}=\sum_{n \geq 1} \mathrm{~A}_{n}(\mathcal{F}) q^{n}
$$

denote the formal $q$-adic expansion of $\mathcal{F}$ with $\mathrm{A}_{n}(\mathcal{F}) \in \mathbb{H}_{\mathcal{F}}^{\text {ord }}$. By a celebrated result of Hida (cf. [Hi], [Wi]) there exists a 'large' continuous irreducible representation

$$
\rho: G_{\mathbb{Q}} \longrightarrow \operatorname{Aut}_{\mathbb{H}_{\mathcal{F}}^{\text {ord }}}\left(\mathcal{T}_{\mathcal{F}}\right)
$$

where $\mathcal{T}_{\mathcal{F}}$ is a finitely generated, torsion-free module of generic rank 2 over $\mathbb{H}_{\mathcal{F}}^{\text {ord }}$. This representation $\rho$ is unramified at all the primes $l \in \mathbb{Z}$ not lying below $S$. Moreover, the trace of the (geometric) Frobenius $\operatorname{Fr}_{l} \in G_{\mathbb{Q}}$ acting on $\mathcal{T}_{\mathcal{F}}$ is equal to the Fourier coefficient $\mathrm{A}_{l}(\mathcal{F})$ of $\mathcal{F}$ for every prime ideal $l$ not lying below $S$.

Let $\mathfrak{m}$ be the maximal ideal of $\mathbb{H}_{\mathcal{F}}^{\text {ord }}$ and let $\mathbb{F}$ denote the finite residue field $\mathbb{H}_{\mathcal{F}}^{\text {ord }} / \mathfrak{m}$. Then we obtain the residual representation

$$
\bar{\rho}: G_{\mathbb{Q}} \longrightarrow \operatorname{Aut}_{\mathbb{H}_{\mathcal{F}}^{\text {ord }}}\left(\mathcal{T}_{\mathcal{F}} / \mathfrak{m}\right)
$$

The representation space of $\bar{\rho}$ is an $\mathbb{F}$-vector space of dimension two with semi-simple $G_{\mathbb{Q}^{-}}$-action, along with the additional property that the trace of $\operatorname{Fr}_{l}$ is congruent to $\mathrm{A}_{l}(\mathcal{F})$ modulo $\mathfrak{m}$, for every prime $l$ not lying below $S$. (see [M-W]) Throughout this article, we shall always assume that the following conditions hold, although we may not always state them explicitly:

(Nor): The ring $\mathbb{H}_{\mathcal{F}}^{\text {ord }} \cong O\left[\left[\Gamma^{\prime}\right]\right]$, where $O$ is some finite extension of $\mathbb{Z}_{p}$.

(Irr): The residual representation $\bar{\rho}$ of $\mathcal{T}_{\mathcal{F}}$ in (3) is an absolutely irreducible $G_{\mathbb{Q}^{-}}$ 
module.

For a non negative integer $w$, recall that an element $\xi \in \operatorname{Hom}_{\mathbb{Z}_{p}}\left(\mathbb{H}_{\mathcal{F}}^{\text {ord }}, \overline{\mathbb{Q}}_{p}\right)$ is called an arithmetic point of weight $w$ if there exists an open subgroup $U$ of $\Gamma^{\prime}$ such that the restriction

$$
\xi_{\left.\right|_{U}}: U \hookrightarrow \mathbb{Z}_{p}\left[\left[\Gamma^{\prime}\right]\right]^{*} \hookrightarrow\left(\mathbb{H}_{\mathcal{F}}^{\text {ord }}\right)^{*} \stackrel{\xi}{\longrightarrow} \overline{\mathbb{Q}}_{p}^{*}
$$

sends $u$ to $\kappa^{w}(u)$, for any $u \in U$. The set of arithmetic points is denoted by $\mathfrak{X}_{\text {arith }}\left(\mathbb{H}_{\mathcal{F}}^{\text {ord }}\right)$, and for an arithmetic point $\xi$, the weight of $\xi$ will be denoted by $w(\xi)$. For any $\xi$ in $\mathfrak{X}_{\text {arith }}\left(\mathbb{H}_{\mathcal{F}}^{\text {ord }}\right)$, put $P_{\xi}=\operatorname{Ker} \xi$. Then $P_{\xi}=\left(p_{\xi}\right)$ is a height 1 principal prime ideal in $\mathbb{H}_{\mathcal{F}}^{\text {ord }}$. In fact, the ideal $\left(p_{\xi}\right)$ in $\mathbb{H}_{\mathcal{F}}^{\text {ord }}$ lies above the prime ideal $(1+p)^{w(\xi)}-(1+T)$ in $\mathbb{Z}_{p}\left[\left[\Gamma^{\prime}\right]\right] \cong \mathbb{Z}_{p}[[T]]$.

Under the assumption (Irr) above, we have the following basic properties (see [Oc, section 2]) :

(i) The module $\mathcal{T}=\mathcal{T}_{\mathcal{F}}$ is a free $\mathbb{H}_{\mathcal{F}}^{\text {ord }}$-module of rank 2 .

(ii) For each $\xi \in \mathfrak{X}_{\text {arith }}\left(\mathbb{H}_{\mathcal{F}}^{\text {ord }}\right)$, there exists a normalized cuspidal eigenform $f_{\xi}$ of weight $w(\xi)+2$, such that the quotient $\mathcal{T} /\left(p_{\xi}\right)$ is isomorphic to $T_{f_{\xi}}$, where $T_{f_{\xi}}$ is the (unique) lattice of Deligne's Galois representation associated to the modular form $f_{\xi}$. We have $T_{f_{\xi}} \cong O_{\xi} \oplus O_{\xi}$, where $O_{\xi}:=\mathbb{H}_{\mathcal{F}}^{\text {ord }} /\left(p_{\xi}\right)$.

(iii) As a representation of the decomposition group $G_{\mathbb{Q}_{p}} \subset G_{\mathbb{Q}}$ at $p, \mathcal{T}=\mathcal{T}_{\mathcal{F}}$ has a filtration

$$
0 \longrightarrow F^{+} \mathcal{T} \longrightarrow \mathcal{T} \longrightarrow F^{-} \mathcal{T} \longrightarrow 0
$$

such that the graded pieces $F^{+} \mathcal{T}$ and $F^{-} \mathcal{T}$ are free of rank 1 over $\mathbb{H}_{\mathcal{F}}^{\text {ord }}$. Moreover $\mathrm{A}_{p}(\mathcal{F}) \in \mathbb{H}_{\mathcal{F}}^{\text {ord }}$ satisfies the interpolation property

$$
\xi\left(\mathrm{A}_{p}(\mathcal{F})\right)=a_{p}\left(f_{\xi}\right),
$$

the $p^{\text {th }}$ Fourier coefficient of the cuspidal ordinary form $f_{\xi}$, for each $\xi \in \mathfrak{X}_{\text {arith }}\left(\mathbb{H}_{\mathcal{F}}^{\text {ord }}\right)$.

Throughout this article we will consider the Galois representation associated to the family of $p$-ordinary, $p$-stabilized newforms that arise as the specialization at arithmetic points of an ordinary $\Lambda$-adic newform $\mathcal{F}$ in the sense discussed above. This will correspond to a branch of a minimal Hida family. In particular, every cuspform in the family has level $N p^{r}$ for some $r$ and their residual representations are all isomorphic. (see [E-P-W] for details.)

2. Fine Selmer group. Let $f=\sum a_{n} q^{n} \in S_{k}\left(\Gamma_{0}\left(N p^{r}\right), \psi\right)$ be a normalized eigenform of weight $\geq 2$, tame level $N$ and character $\psi$. We further assume that $f$ is ordinary at $p$, and that $f$ is $p$-stabilized. Let $K_{f}$ be the number field generated by the Fourier coefficients of $f$ and the values of $\psi$. Let $K_{f, v}$ denote the completion of this number field at a prime $v$ lying above $p$ and let $O_{f}$ denote its ring of integers. By results of Deligne, Eichler, Shimura, and Mazur-Wiles, Wiles (cf. [Wi]) there exists a Galois representation

$$
\rho_{f}: \mathrm{G}_{\mathbb{Q}} \longrightarrow \mathrm{GL}_{2}\left(K_{f, v}\right)
$$

which is ordinary at $p$, and unramified at all primes $l$ not dividing $N p$. Let $V_{f}$ denote the representation space of $\rho_{f}$. Choose a lattice $T_{f}$ of $V_{f}$ which is invariant under $\rho_{f}$, so that we get an action of $G_{\mathbb{Q}}$ on $A_{f}=V_{f} / T_{f}$. Let

$$
\bar{\rho}: \mathrm{G}_{\mathbb{Q}} \longrightarrow \mathrm{GL}_{2}\left(O_{f} / \pi\right)
$$


be the residual representation of $\rho$, where $\pi$ is a uniformizing parameter for $K_{f, v}$. If we further assume that $\bar{\rho}_{f}$ is an absolutely irreducible representation of $G_{\mathbb{Q}}$, then there exists a unique (up to conjugation) Galois invariant lattice in $V_{f}$ (see, for example $[\mathrm{Ca}])$.

For a finite extension $L$ of $K$ and each each $v$ in $S$, we define $K_{v}^{i}\left(A_{f} / L\right)$ as

$$
K_{v}^{i}\left(A_{f} / L\right):=\underset{w \mid v}{\oplus} H^{i}\left(L_{w}, A_{f}\right)
$$

for $i=0,1,2$, where for each prime $w$ in $L$ lying over $v, L_{w}$ denotes a completion of $L$ at $w$. For an infinite extension $L_{\infty}$ of $K$, the groups $K_{v}^{i}\left(A_{f} / L_{\infty}\right)$ are defined by taking the inductive limit of $K_{v}^{i}\left(A_{f} / L^{\prime}\right)$ over all finite extensions $L^{\prime}$ of $K$ contained in $L_{\infty}$. Let $\mathcal{L}$ be a $p$-adic Lie extension of $K$ and $S$ be chosen as before. We now define the fine Selmer group of $f$ over $\mathcal{L} / K$ for the lattice $T_{f}$, denoted by $R\left(A_{f} / \mathcal{L}\right)$ as

Definition 1. $R\left(A_{f} / \mathcal{L}\right)=\operatorname{Ker}\left(H^{1}\left(K_{S} / \mathcal{L}, A_{f}\right) \longrightarrow \underset{v \in S}{\oplus} K_{v}^{1}\left(A_{f} / \mathcal{L}\right)\right)$.

We denote the Pontryagin dual $R\left(A_{f} / \mathcal{L}\right)^{\vee}$ by $Y\left(T_{f} / \mathcal{L}\right)$. Then $Y\left(T_{f} / \mathcal{L}\right)$ is a finitely generated (left) module over the Iwasawa algebra $O_{f}[[\mathcal{G}]]$. Since $K / \mathbb{Q}$ is abelian, by a deep result of Kato (see $[\mathrm{Ka}]$ ), the 'Weak Leopoldt Conjecture' holds for $K_{\text {cyc }}$ i.e.

$$
H^{2}\left(K_{S} / K_{\mathrm{cyc}}, A_{f}\right)=0 .
$$

It then follows (see for example [C-S, Lemma 3.1]) that $Y\left(T_{f} / K_{\text {cyc }}\right)$ is a finitely generated torsion $\Lambda_{O_{f}}[[\Gamma]]$ module.

Let $f$ be a cuspidal eigenform as in the begining of this section. The generalization of Conjecture 1 in $[\mathrm{C}-\mathrm{S}]$ is the following conjecture:

Conjecture A. $Y\left(T_{f} / K_{\text {cyc }}\right)$ is a finitely generated $O_{f}$-module.

To proceed further we need the following lemmas. The first lemma is a generalization of a result of Howson [Ho1, Theorem 1.1]. The proof is similar and hence we skip the proof.

LEMMA 1. Let $B$ be a complete, commutative, regular local ring with finite residue field, and let $M$ be a finitely generated $B[[\mathcal{H}]]$-module. Then for all $i$, the groups $H_{i}(\mathcal{H}, M)$ are finitely generated $B$-modules and we have

$$
\operatorname{rank}_{B[[\mathcal{H}]]} M=\sum_{i \geq 0}(-1)^{i} \operatorname{rank}_{B} H_{i}(\mathcal{H}, M) .
$$

We use the above lemma to prove the following generalized 'fundamental diagram'.

Lemma 2. Let $\mathcal{L}, \mathcal{H}, S$ and $B$ are as defined before. Take $C$ to be a cofinitely generated $B$-module with continuous $G_{\mathbb{Q}}$ action such that the action is trivial when restricted to the subgroup $\operatorname{Gal}\left(\overline{\mathbb{Q}} / K_{S}\right)$. Then

(i) the kernel and the cokernel of the map

$$
H^{1}\left(K_{S} / K_{\text {cyc }}, C\right) \stackrel{\alpha}{\longrightarrow} H^{1}\left(K_{S} / \mathcal{L}, C\right)^{\mathcal{H}}
$$

are cofinitely generated B-modules.

(ii) for each $v$ in $S$, the kernel of the map

$$
K_{v}^{1}\left(C / K_{\text {cyc }}\right) \stackrel{\beta_{v}}{\longrightarrow} K_{v}^{1}(C / \mathcal{L})^{\mathcal{H}}
$$


is a cofinitely generated $B$-module, where $K_{v}^{i}(C / \mathcal{L})$ is defined by replacing $A_{f}$ by $C$ in (7).

Proof. The kernel of the map $\alpha$ is $H^{1}\left(\mathcal{H}, C^{G_{\mathcal{L}}}\right)$ and cokernel of $\alpha$ is contained in $H^{2}\left(\mathcal{H}, C^{G_{\mathcal{L}}}\right)$. Since $C$ itself is a cofinitely generated $B$-module, it is obvious that $C^{G_{\mathcal{L}}}$ is a cofinitely generated $B[[\mathcal{H}]]$-module. It follows from Lemma 1 that $H^{i}\left(\mathcal{H}, C^{G_{\mathcal{L}}}\right)^{\vee} \cong H_{i}\left(\mathcal{H},\left(C^{G_{\mathcal{L}}}\right)^{\vee}\right)$ is a finitely generated $B$-module, which proves (i). To prove (ii), we see that, using Shapiro's Lemma, the kernel of $\beta_{v}$ is isomorphic to $\underset{w \mid v}{\oplus} H^{1}\left(\mathcal{H}_{w}, C^{G_{\mathcal{L}_{w}}}\right)$, where $w$ runs over the finite set of primes of $K_{\text {cyc }}$ lying above $v$,

and $\mathcal{H}_{w}$ denotes a decomposition group in $\mathcal{H}$ of some fixed extension of $w$ to $\mathcal{L}$. Now proceeding as in (i) and replacing $\mathcal{H}$ and $\mathcal{L}$ respectively by their local counterparts $\mathcal{H}_{w}$ and $\mathcal{L}_{w}$ and summing over finitely many $w$, we get (ii). Thus the Lemma is proved. $\mathbf{\square}$

Assume Conjecture A holds for $Y\left(T_{f} / K_{\text {cyc }}\right)$. Then putting $B=O_{f}$ and $C=A_{f}$ in Lemma 2 and using the definition of $R\left(A_{f} / \mathcal{L}\right)$, we deduce that the kernel and the cokernel of the map

$$
Y\left(T_{f} / \mathcal{L}\right)_{\mathcal{H}} \longrightarrow Y\left(T_{f} / K_{\text {cyc }}\right)
$$

are finitely generated $O_{f}$ modules and hence using Nakayama's Lemma we see that $Y\left(T_{f} / \mathcal{L}\right)$ is a finitely generated $\Lambda_{O_{f}}(\mathcal{H})$ module.

Let $B$ and $\mathcal{G}$ be as before. Let $M$ be a finitely generated torsion $B[[\mathcal{G}]]$-module. We recall that $M$ is defined to be a pseudonull $B[[\mathcal{G}]]$-module if $\operatorname{Ext}_{B[[\mathcal{G}]]}^{1}(M, B[[G]])=0$. In the case $B=O$, a finite extension of $\mathbb{Z}_{p}$, a result of Venjakob [Ve] asserts that an $O[[\mathcal{G}]]$-module which is a finitely generated $O[[\mathcal{H}]]$-module is pseudonull as an $O[[\mathcal{G}]]$ module if and only if it is a torsion $O[[\mathcal{H}]]$-module. Now we extend Conjecture B of [C-S] as follows.

Conjecture B. Assume Conjecture A holds for $Y\left(T_{f} / K_{\mathrm{cyc}}\right)$. Let dimension of $\mathcal{G}$ as a $p$-adic Lie group is $\geq 2$. Then the fine Selmer group $Y\left(T_{f} / \mathcal{L}\right)$ is a finitely generated torsion $\Lambda_{O_{f}}(\mathcal{H})$ module or equivalently a pseudonull $\Lambda_{O_{f}}(\mathcal{G})$ module.

Next we define the fine Selmer group associated to the large Galois representation (2). The discrete dual of $\mathcal{T}$ is given by

$$
\mathcal{A}=\mathcal{T} \otimes_{\mathbb{H}_{\mathcal{F}} \text { ord }} \operatorname{Hom}_{\mathbb{Z}_{p}}\left(\mathbb{H}_{\mathcal{F}}^{\text {ord }}, \mathbb{Q}_{p} / \mathbb{Z}_{p}\right) .
$$

Let $S$ be chosen as before and let $K_{v}^{i}(\mathcal{A} / \mathcal{L})$ be defined as in $(7)$ with $A_{f}$ being replaced by $\mathcal{A}$. The fine Selmer group of the $\Lambda$-adic representation, over the $p$-adic Lie extension $\mathcal{L}$ of $K$ denoted by $\mathcal{R}(\mathcal{A} / \mathcal{L})$, is defined by

$$
\mathcal{R}(\mathcal{A} / \mathcal{L})=\operatorname{Ker}\left(H^{1}\left(K_{S} / \mathcal{L}, \mathcal{A}\right) \longrightarrow \underset{v \in S}{\oplus} K_{v}^{1}(\mathcal{A} / \mathcal{L})\right) .
$$

Clearly $\mathcal{R}(\mathcal{A} / \mathcal{L})$ is an $\mathbb{H}_{\mathcal{F}}^{\text {ord }}$-module. Further, the action of the Galois group $\mathcal{G}$ on the cohomology groups commutes with the $\mathbb{H}_{\mathcal{F}}^{\text {ord }}$-action, thereby endowing $\mathcal{R}(\mathcal{A} / \mathcal{L})$ with a structure of an $\mathbb{H}_{\mathcal{F}}^{\text {ord }}[[\mathcal{G}]]$-module. The corresponding Pontryagin dual in this case is denoted by $\mathcal{Y}(\mathcal{T} / \mathcal{L})$.

Since $K / \mathbb{Q}$ is abelian, it can be shown (see [Oc, Proposition 4.9]) that $\mathcal{Y}\left(\mathcal{T} / K_{\text {cyc }}\right.$ ) is a finitely generated torsion $\mathbb{H}_{\mathcal{F}}^{\text {ord }}[[\Gamma]]$-module. The following is a generalization of Conjecture 1 of [J-S] to an arbitrary abelian number field $K$,

Conjecture 1. $\mathcal{Y}\left(\mathcal{T} / K_{\text {cyc }}\right)$ is a finitely generated $\mathbb{H}_{\mathcal{F}}^{\text {ord }}$-module. 
For a $B$-module $M$ and an element $\alpha \in B$, define $M[\alpha]=\{x \in M: \alpha x=0\}$. Using the fact that $\mathcal{T} / p_{\xi} \cong T_{f_{\xi}}$, it is immediate that $\mathcal{A}\left[p_{\xi}\right] \cong A_{f_{\xi}}$, where $f_{\xi}$ is the cuspform associated to the specialization of $\mathcal{T}$ at the arithmetic point $\xi$. The following theorem establishes the relation between Conjecture A and Conjecture 1.

THEOREM 3. The following are equivalent.

1. There exist $\xi_{0} \in \mathfrak{X}_{\text {arith }}\left(\mathbb{H}_{\mathcal{F}}^{\text {ord }}\right)$ such that Conjecture $A$ is true for $Y\left(T_{f_{\xi_{0}}} / K_{\mathrm{cyc}}\right)$.

2. For every $\xi \in \mathfrak{X}_{\text {arith }}\left(\mathbb{H}_{\mathcal{F}}^{\text {ord }}\right)$, Conjecture $A$ is true for $Y\left(T_{f_{\xi}} / K_{\text {cyc }}\right)$.

3. Conjecture 1 is true for $\mathcal{Y}\left(\mathcal{T} / K_{\text {cyc }}\right)$.

Proof. The proof is essentially same as that of [J-S, Theorem 8] and hence we skip the proof.

Assume that Conjecture 1 holds for $\mathcal{Y}\left(\mathcal{T} / K_{\text {cyc }}\right)$. Now setting $B=\mathbb{H}_{\mathcal{F}}^{\text {ord }}$ and $C=\mathcal{A}$ in Lemma 2 and using the definition of $\mathcal{R}(\mathcal{A} / \mathcal{L})$, we deduce that the kernel and the cokernel of the map

$$
\mathcal{Y}(\mathcal{T} / \mathcal{L})_{\mathcal{H}} \longrightarrow \mathcal{Y}\left(\mathcal{T} / F_{\text {cyc }}\right)
$$

are finitely generated $\mathbb{H}_{\mathcal{F}}^{\text {ord }}$-modules and hence using Nakayama's Lemma, we get that $\mathcal{Y}(\mathcal{T} / \mathcal{L})$ is a finitely generated $\mathbb{H}_{\mathcal{F}}^{\text {ord }}[[\mathcal{H}]]$-module. We propose the following conjecture:

Conjecture 2. Assume that Conjecture 1 is true for $\mathcal{Y}\left(\mathcal{T} / K_{\text {cyc }}\right)$. Let dimension of $\mathcal{G}$ as a $p$-adic Lie group is $\geq 2$. Then $\mathcal{Y}(\mathcal{T} / \mathcal{L})$ is a finitely generated torsion $\mathbb{H}_{\mathcal{F}}^{\text {ord }}[[\mathcal{H}]]$-module.

We shall study the relation between Conjecture B and Conjecture 2 in section 3.

3. Control theorem and specializations. Recall from the previous section that for an arithmetic point $\xi$, we have $\mathcal{A}\left[p_{\xi}\right] \cong A_{f_{\xi}}$, the discrete lattice associated to the eigenform $f_{\xi}$. There is an obvious map

$$
R\left(\mathcal{A}\left[p_{\xi}\right] / \mathcal{L}\right) \stackrel{r_{\xi}}{\longrightarrow} \mathcal{R}(\mathcal{A} / \mathcal{L})\left[p_{\xi}\right] .
$$

Our aim is to prove a "control theorem" for the above maps.

Theorem 4. Assume that the conditions (Nor) and (Irr) hold. Then the Pontryagin dual of the kernel and the cokernel of the map

$$
R\left(\mathcal{A}\left[p_{\xi}\right] / \mathcal{L}\right) \stackrel{r_{\xi}}{\longrightarrow} \mathcal{R}(\mathcal{A} / \mathcal{L})\left[p_{\xi}\right]
$$

are finitely generated $O_{\xi}[[\mathcal{H}]]$-modules for all $\xi \in \mathfrak{X}_{\text {arith }}\left(\mathbb{H}_{\mathcal{F}}^{\text {ord }}\right)$ and are even $O_{\xi}[[\mathcal{H}]]-$ torsion i.e. $O_{\xi}[[\mathcal{G}]]$-pseudonull for all but finitely many such $\xi$.

Proof. Consider the following commutative diagram

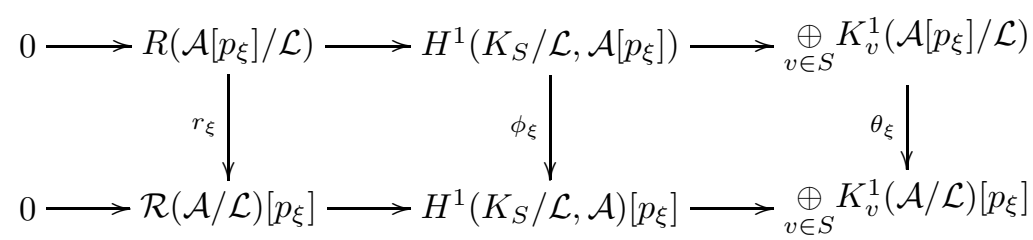

where all the vertical arrows are the natural maps. It is plain from the Kummer sequence that the middle vertical arrow is surjective. Now the kernel of the middle 
vertical map is $\left(\mathcal{A}^{\mathcal{G}_{\infty}}\right) / p_{\xi}$, where $\mathcal{G}_{\infty}$ denotes the Galois group $\operatorname{Gal}(\overline{\mathbb{Q}} / \mathcal{L})$. Hence $\left(\text { Ker } r_{\xi}\right)^{\vee}$ is isomorphic to a quotient of $\left(\mathcal{T}^{*}\right)_{\mathcal{G}_{\infty}}\left[p_{\xi}\right]$, where $\mathcal{T}^{*}$ denotes the module $\operatorname{Hom}_{\mathbb{H}} \mathbb{H o r d}_{\mathcal{F}}\left(\mathcal{T}, \mathbb{H}_{\mathcal{F}}^{\text {ord }}\right)$. Clearly $\mathcal{T}^{*}$ is a finitely generated $\mathbb{H}_{\mathcal{F}}^{\text {ord }}$-module, and hence $\mathcal{T}^{*}\left[p_{\xi}\right]$ is a finitely generated $\mathbb{H}_{\mathcal{F}}^{\text {ord }} / p_{\xi} \cong O_{\xi}$-module, whence a finitely generated torsion $O_{\xi}[[\mathcal{H}]]-$ module. Thus we have shown that $\left(\operatorname{Ker} r_{\xi}\right)^{\vee}$ is a finitely generated torsion $O_{\xi}[[\mathcal{H}]]$-module for all $\xi \in \mathfrak{X}_{\text {arith }}\left(\mathbb{H}_{\mathcal{F}}^{\text {ord }}\right)$.

Next we show that $\left(\text { Coker } r_{\xi}\right)^{\vee}$ is a finitely generated $O_{\xi}[[\mathcal{H}]]$-module for all $\xi \in$ $\mathfrak{X}_{\text {arith }}\left(\mathbb{H}_{\mathcal{F}}^{\text {ord }}\right)$. Clearly it suffices to show that $\left(\operatorname{Ker} \theta_{\xi}\right)^{\vee}$ is a finitely generated $O_{\xi}[[\mathcal{H}]]-$ module for all $\xi$. Now using Shapiro's lemma, it is easy to see that Ker $\theta_{\xi} \cong$ $\left(\underset{v \in S}{\oplus} K_{v}^{0}(\mathcal{A} / \mathcal{L})\right) / p_{\xi}$. For each $v$, and for any extension $L$ of $K$, set $\mathcal{U}_{v}^{L}:=\left(K_{v}^{0}(\mathcal{A} / L)\right)^{\vee}$. Then again using Shapiro's lemma, it follows that $\left(\mathcal{U}_{v}^{\mathcal{L}}\right)_{\mathcal{H}} \cong \mathcal{U}_{v}^{K_{\text {cyc }}}$. Now there are only finitely many primes in $K_{\text {cyc }}$ lying over a prime $v$ of $K$. Using this it is easy to see that $\mathcal{U}_{v}^{K_{\text {cyc }}}$ is a finitely generated $\mathbb{H}_{\mathcal{F}}^{\text {ord }}$-module. Hence by Nakayama's lemma, it is immediate that $\mathcal{U}_{v}^{\mathcal{L}}$ is finitely generated $\mathbb{H}_{\mathcal{F}}^{\text {ord }}[[\mathcal{H}]]$-module. Thus $\mathcal{U}_{v}^{\mathcal{L}}\left[p_{\xi}\right]$ is a finitely generated $O_{\xi}[[\mathcal{H}]]$-module. Summing over finitely many $v \in S$, we get that $\left(\operatorname{Ker} \theta_{\xi}\right)^{\vee}$ is a finitely generated $O_{\xi}[[\mathcal{H}]]$-module.

Now we go on to show that $\left(\operatorname{Ker} \theta_{\xi}\right)^{\vee}$ (and hence $\left(\text { Coker } r_{\xi}\right)^{\vee}$ ) is a finitely generated torsion $O_{\xi}[[\mathcal{H}]]$-module for all but finitely many $\xi \in \mathfrak{X}_{\text {arith }}\left(\mathbb{H}_{\mathcal{F}}^{\text {ord }}\right)$. We need the following lemma to complete the proof. Recall that each $p_{\xi}$, lies in the centre of $\mathbb{H}_{\mathcal{F}}$ ord $[[\mathcal{H}]]$ and $\mathbb{H}_{\mathcal{F}}^{\text {ord }}[[\mathcal{H}]] / p_{\xi} \cong O_{\xi}[[\mathcal{H}]]$.

Lemma 5. Let $N$ be a finitely generated $\mathbb{H}_{\mathcal{F}}^{\text {ord }}[[\mathcal{H}]]$-module. Then

$$
\operatorname{rank}_{O_{\xi}[[\mathcal{H}]]} N / p_{\xi}=\operatorname{rank}_{\left.\mathbb{H} \text { ord }_{\mathcal{F}}[\mathcal{H}]\right]} N+\operatorname{rank}_{O_{\xi}[[\mathcal{H}]]} N\left[p_{\xi}\right] .
$$

Proof. We use the homological definition of rank of $N$ as $\mathbb{H}_{\mathcal{F}}^{\text {ord }}[[\mathcal{H}]]$-module and use the fact $\operatorname{Tor}_{\mathbb{H}_{\mathcal{F}} \text { ord }[[\mathcal{H}]]}^{1}\left(N, \mathbb{H}_{\mathcal{F}}^{\text {ord }}[[\mathcal{H}]] / p_{\xi}\right) \cong N\left[p_{\xi}\right]$. From these two facts the lemma follows easily.

We continue the proof of the Theorem 4. Let us denote $\mathbb{H}_{\mathcal{F}}^{\text {ord }}[[\mathcal{H}]]$ by $R$ and $\underset{v \in S}{\oplus} \mathcal{U}_{v}^{\mathcal{L}}$ by $M$ for the rest of the proof. Then we have to show that

$$
\text { for all but finitely many } \xi, \operatorname{rank}_{R / p_{\xi}} M\left[p_{\xi}\right]=0 .
$$

Recall that an element $l \in R$ is called a central prime if $l$ is a prime in $R$ and lies in the centre of $R$. Let $M_{\mathrm{ct}}$ be the submodule of $M$ generated by elements of $M$ annihilated by any product of central primes. By definition of $M_{\mathrm{ct}}$, we get that for any $\xi,\left(M / M_{\mathrm{ct}}\right)\left[p_{\xi}\right]=0$. Thus applying Lemma 5 with $N=M / M_{\mathrm{ct}}$ we get the assertion that

$$
\text { for all } \xi \in \mathfrak{X}_{\text {arith }}\left(\mathbb{H}_{\mathcal{F}}^{\text {ord }}\right), \operatorname{rank}_{R / p_{\xi}}\left(M / M_{\mathrm{ct}}\right) / p_{\xi}=\operatorname{rank}_{R} M / M_{\mathrm{ct}} .
$$

Also we have

$$
\operatorname{rank}_{R} M=\operatorname{rank}_{R} M / M_{\mathrm{ct}} .
$$

Using (16) in Lemma 5 for $N=M$, it is immediate that

(17) for all $\xi \in \mathfrak{X}_{\text {arith }}\left(\mathbb{H}_{\mathcal{F}}^{\text {ord }}\right), \operatorname{rank}_{R / p_{\xi}} M / p_{\xi}=\operatorname{rank}_{R} M / M_{\text {ct }}+\operatorname{rank}_{R / p_{\xi}} M\left[p_{\xi}\right]$. 
Note that $R=\mathbb{H}_{\mathcal{F}}^{\text {ord }}[[\mathcal{H}]] \cong O\left[\left[G^{\prime}\right]\right]$ where $O$ is the ring of integers of a $p$-adic number field and $G^{\prime} \cong \Gamma \times \mathcal{H}$. With this identification it follows from [Ho2, Theorem 2.5] that there exist a pseudoisomorphism $\phi$,

$$
0 \rightarrow P_{1} \longrightarrow M \stackrel{\phi}{\longrightarrow} \underset{1 \leq i \leq n}{\oplus} \frac{O\left[\left[G^{\prime}\right]\right]}{\left(q_{i}^{n_{i}}\right)} \oplus M / M_{\mathrm{ct}} \longrightarrow P_{2} \rightarrow 0
$$

where $q_{i}$ for $i=1, \ldots, n$ are central primes and $P_{1}, P_{2}$ are pseudonull $R$ modules. This representation is unique upto a permutation of $q_{1}, \ldots, q_{n}$. Now for any $p_{\xi}$ not in the set $\left\{q_{1}, \ldots, q_{n}\right\}$, it follows that $\frac{O\left[\left[G^{\prime}\right]\right]}{\left(q_{i}^{n_{i}}\right)} / p_{\xi}$ is $R / p_{\xi}$-torsion, so that $\operatorname{rank}_{R / p_{\xi}}\left(\underset{1 \leq i \leq n}{\oplus} \frac{O\left[\left[G^{\prime}\right]\right]}{\left(q_{i}^{n^{*}}\right)} \oplus\right.$ $\left.M / M_{\mathrm{ct}}\right) / p_{\xi}=\operatorname{rank}_{R / p_{\xi}}\left(M / M_{\mathrm{ct}}\right) / p_{\xi}$. Also for a pseudonull $R$ module $P$, both $P\left[p_{\xi}\right]$ and $P / p_{\xi}$ are torsion as $R / p_{\xi}$ modules. This follows from the fact that $E_{R / p_{\xi}}^{0}\left(P\left[p_{\xi}\right]\right) \cong$ $E_{R}^{1}\left(P\left[p_{\xi}\right]\right)=0$ and similarly $E_{R / p_{\xi}}^{0}\left(P / p_{\xi}\right) \cong E_{R}^{1}\left(P / p_{\xi}\right)=0$. We split (18) in to two short exact sequences and tensor each of them with $R / p_{\xi}$ (over $R$ ). Using the above observations and noting that alternating rank is zero in an exact sequence, we get

$$
\text { for all but finitely many } \xi, \operatorname{rank}_{R / p_{\xi}} M / p_{\xi}=\operatorname{rank}_{R / p_{\xi}}\left(M / M_{\mathrm{ct}}\right) / p_{\xi} \text {. }
$$

Hence using (19) and (15) in (17) we deduce that for all but finitely many $\xi \in$ $\mathfrak{X}_{\text {arith }}\left(\mathbb{H}_{\mathcal{F}}^{\text {ord }}\right)$, we have

$$
\operatorname{rank}_{R / p_{\xi}} M\left[p_{\xi}\right]\left(=\operatorname{rank}_{R / p_{\xi}}\left(M / M_{\mathrm{ct}}\right)\left[p_{\xi}\right]\right)=0,
$$

which establishes (14) and hence the theorem is proved.

REMARK 6. We stress that we do not need to assume Conjecture 1 for proving the control theorem. This is clear from the proof.

REMARK 7. The last part of the proof shows that for a finitely generated $R:=$ $\mathbb{H}_{\mathcal{F}}^{\text {ord }}[[\mathcal{H}]]$-module $M$, the equality

$$
\operatorname{rank}_{R} M=\operatorname{rank}_{R / p_{\xi}} M / p_{\xi}
$$

holds for all but finitely many $\xi$.

Assume now that one of the equivalent conditions in Theorem 3 holds. Then we know that both $\mathcal{Y}(\mathcal{T} / \mathcal{L}) / p_{\xi}$ and $Y\left(T_{\xi} / \mathcal{L}\right)$ are finitely generated modules over $O_{\xi}[[\mathcal{H}]]$ for any $\xi$. By Remark 7 , for all but finitely many $\xi$, the $\mathbb{H}_{\mathcal{F}}^{\text {ord }}[[\mathcal{H}]]$ rank of $\mathcal{Y}(\mathcal{T} / \mathcal{L})$ and the $O_{\xi}[[\mathcal{H}]]$ rank of $\mathcal{Y}(\mathcal{T} / \mathcal{L}) / p_{\xi}$ are the same. Combining these facts with Theorem 4 , we get the following result.

Proposition 8. Assume (Nor), (Irr) and that $K / \mathbb{Q}$ is abelian. Also assume that Conjecture $A$ holds for some $\xi_{0} \in \mathfrak{X}_{\text {arith }}\left(\mathbb{H}_{\mathcal{F}}^{\text {ord }}\right)$. Then for all but finitely many $\xi \in \mathfrak{X}_{\text {arith }}\left(\mathbb{H}_{\mathcal{F}}^{\text {ord }}\right)$, the $O_{\xi}[[\mathcal{H}]]$ ranks of $Y\left(T_{\xi} / \mathcal{L}\right)$ are the same with the rank being equal to the $\mathbb{H}_{\mathcal{F}}^{\text {ord }}[[\mathcal{H}]]$ rank of $\mathcal{Y}(\mathcal{T} / \mathcal{L})$.

Proof. Let $\Sigma$ be the finite subset of $\mathfrak{X}_{\text {arith }}\left(\mathbb{H}_{\mathcal{F}}^{\text {ord }}\right)$ consisting of $\xi$ 's for which the $\mathbb{H}_{\mathcal{F}}^{\text {ord }}[[\mathcal{H}]]$ rank of $\mathcal{Y}(\mathcal{T} / \mathcal{L})$ is strictly less than the $O_{\xi}[[\mathcal{H}]]$ rank of $Y\left(T_{\xi} / \mathcal{L}\right)$ and those $\xi$ for which (Coker $\left.r_{\xi}\right)^{\vee}$ in Theorem 4 is not $O_{\xi}[[\mathcal{H}]]$-torsion. For any $\xi \in \mathbb{H}_{\mathcal{F}}^{\text {ord }} \backslash \Sigma$ the assertion follows directly from Theorem 4 and the above discussions.

We make the following hypothesis for an admissible $p$-adic Lie extension $\mathcal{L}$ of a number field $K$ with $\mathcal{G} \cong \operatorname{Gal}(\mathcal{L} / K)$. Note that since $K_{\text {cyc }} \subset \mathcal{L}$ we know that for each $v$, the decomposition group $\mathcal{G}_{v}$ has dimension $\geq 1$. 
$\left(\operatorname{Dim}_{S}\right)$ : For each $v$ in $S$, The decomposition group of $\mathcal{G}$ at $v$, denoted by $\mathcal{G}_{v}$, has dimension $\geq 2$.

Now we can deduce the following corollary from the above control theorem.

Corollary 9. Assume Conjecture $A$ holds for some $\xi \in \mathfrak{X}_{\text {arith }}\left(\mathbb{H}_{\mathcal{F}}^{\text {ord }}\right)$ and also $\left(\operatorname{Dim}_{S}\right)$ holds. Then for every $\xi \in \mathfrak{X}_{\text {arith }}\left(\mathbb{H}_{\mathcal{F}}^{\text {ord }}\right)$, the $O_{\xi}[[\mathcal{H}]]$ rank of $\mathcal{Y}(\mathcal{T} / \mathcal{L}) / p_{\xi}$ and $Y\left(T_{\xi} / \mathcal{L}\right)$ are the same.

Proof. We continue to use the notation introduced in Theorem 4. First note that, the corollary follows from the proof of control theorem once we prove that for every $\xi \in \mathfrak{X}_{\text {arith }}\left(\mathbb{H}_{\mathcal{F}}^{\text {ord }}\right), \operatorname{rank}_{O_{\xi}[[\mathcal{H}]]}\left(\operatorname{Ker} \theta_{\xi}\right)^{\vee}=0$ i.e. $\operatorname{rank}_{R / p_{\xi}} \underset{v \in S}{\oplus} \mathcal{U}_{v}^{\mathcal{L}}\left[p_{\xi}\right]=0$. Set, as before, $M=\underset{v \in S}{\oplus} \mathcal{U}_{v}^{\mathcal{L}}$. We will show that for all $\xi, \operatorname{rank}_{R / p_{\xi}} M / p_{\xi}=0$. Then $\operatorname{rank}_{R / p_{\xi}} M\left[p_{\xi}\right]=0$ will follow immediately from Lemma 5. Fix a prime in $\mathcal{L}$ above $v$ (abusing notation we continue to denote it by $v$ ) and set $\mathcal{W}_{v}^{\mathcal{L}}:=\left(H^{0}\left(G_{\mathcal{L}_{v}}, \mathcal{A}\right)\right)^{\vee}$. Then we have

$$
\mathcal{U}_{v}^{\mathcal{L}} \cong \mathbb{H}_{\mathcal{F}}^{\text {ord }}[[\mathcal{G}]] \otimes_{\mathbb{H}_{\mathcal{F}}^{\text {ord }}\left[\left[\mathcal{G}_{v}\right]\right]} \mathcal{W}_{v}^{\mathcal{L}}
$$

Similarly, we see that

$$
\mathcal{U}_{v}^{\mathcal{L}} / p_{\xi} \cong O_{\xi}[[\mathcal{G}]] \otimes_{O_{\xi}\left[\left[\mathcal{G}_{v}\right]\right]} W_{\xi, v}^{\mathcal{L}}
$$

where $W_{\xi, v}^{\mathcal{L}}:=\left(H^{0}\left(G_{\mathcal{L}_{v}}, A_{f_{\xi}}\right)\right)^{\vee}$. By $\left(\operatorname{Dim}_{S}\right)$, it follows that dimension of $\mathcal{H}_{v} \geq 1$. Now $W_{\xi, v}^{\mathcal{L}}$, being a finitely generated $\mathbb{Z}_{p}$-module, is torsion as an $O_{\xi}\left[\left[\mathcal{H}_{v}\right]\right]$-module and hence pseudonull as an $O_{\xi}\left[\left[\mathcal{G}_{v}\right]\right]$-module. Note that

$$
\operatorname{Ext}_{O_{\xi}[[\mathcal{G}]]}^{1}\left(\mathcal{U}_{v}^{\mathcal{L}} / p_{\xi}, O_{\xi}[[G]]\right) \cong O_{\xi}[[\mathcal{G}]] \otimes_{O_{\xi}\left[\left[\mathcal{G}_{v}\right]\right]} \operatorname{Ext}_{O_{\xi}\left[\left[\mathcal{G}_{v}\right]\right]}^{1}\left(W_{\xi, v}^{\mathcal{L}}, O_{\xi}\left[\left[\mathcal{G}_{v}\right]\right]\right)
$$

Consequently, we see that $\mathcal{U}_{v}^{\mathcal{L}} / p_{\xi}$ is pseudonull as an $O_{\xi}[[\mathcal{G}]]$-module and hence a finitely generated torsion $O_{\xi}[[\mathcal{H}]]$-module for all $\xi$. Thus $\operatorname{rank}_{R / p_{\xi}} \mathcal{U}_{v}^{\mathcal{L}} / p_{\xi}=0$ holds for all $\xi$. Summing over finitely many $v \in S$, we get that $\operatorname{rank}_{R / p_{\xi}} M / p_{\xi}=0$ for all $\xi \in \mathbb{H}_{\mathcal{F}}^{\text {ord }}$. Hence the corollary is proved.

We now discuss the invariance of the pseudonullity conjecture in a branch of a Hida family.

Theorem 10. Assume (Nor), (Irr), $\left(\mathbf{D i m}_{S}\right)$ and $K / \mathbb{Q}$ is abelian. Let

$$
\rho: \mathrm{G}_{\mathbb{Q}} \longrightarrow \operatorname{Aut}_{\mathbb{H}_{\mathcal{F}} \text { ord }}\left(\mathcal{T}_{\mathcal{F}}\right)
$$

be an ordinary Hida deformation, where $\mathcal{T}=\mathcal{T}_{\mathcal{F}}$ is a finitely generated free module of rank 2 over the ordinary Hecke algebra $\mathbb{H}_{\mathcal{F}}^{\text {ord }}$, associated to a ordinary $\Lambda$-adic newform $\mathcal{F}$. Then the following assertions are equivalent:

1. Conjecture 2 holds for the $\mathbb{H}_{\mathcal{F}}^{\text {ord }}[[\mathcal{G}]]$-module $\mathcal{Y}(\mathcal{T} / \mathcal{L})$.

2. Conjecture B holds for the dual fine Selmer group $Y\left(A_{f_{\xi}} / \mathcal{L}\right)$, for all but finitely many specialization $p_{\xi}$ with $\xi \in \mathfrak{X}_{\text {arith }}\left(\mathbb{H}_{\mathcal{F}}^{\text {ord }}\right)$.

3. There exists $\xi_{0} \in \mathfrak{X}_{\text {arith }}\left(\mathbb{H}_{\mathcal{F}}^{\text {ord }}\right)$ such that Conjecture $B$ holds for $Y\left(A_{f_{\xi_{0}}} / \mathcal{L}\right)$.

Proof. Assume $(1)$ holds. Then $\mathcal{Y}(\mathcal{T} / \mathcal{L})$ is a finitely generated torsion module over $\mathbb{H}_{\mathcal{F}}^{\text {ord }}[[\mathcal{H}]]$ and hence by Proposition 8 we get $(2)$. It is obvious that $(2) \Rightarrow(3)$. To prove $(3) \Rightarrow(1)$, suppose that for some $\xi_{0}$, we have $Y\left(A_{f_{\xi_{0}}} / \mathcal{L}\right)$ is a finitely generated $O_{\xi_{0}}[[\mathcal{H}]]$-torsion module. Since we have assumed $\left(\mathbf{D i m}_{S}\right)$, by Corollary 9 we get that 
$(\mathcal{Y}(\mathcal{T} / \mathcal{L})) / p_{\xi_{0}}$ is $O_{\xi_{0}}[[\mathcal{H}]]$-torsion. Then $(1)$ follows from Lemma 5 , and the theorem is proved.

We now consider the special case when the $p$-adic Lie extension is the false Tate curve extension which we define below. Set $K=\mathbb{Q}\left(\mu_{p}\right)$ and $K_{\text {cyc }}=\mathbb{Q}\left(\mu_{p \infty}\right)$. Let $m$ be a positive integer which is $p$-power free. The false Tate curve extension $K_{\infty}$ over $K$ is defined by

$$
K_{\infty}=\bigcup_{n \geq 1} \mathbb{Q}\left(\mu_{p^{n}}, m^{1 / p^{n}}\right)
$$

Denote by $G=\operatorname{Gal}\left(K_{\infty} / K\right), H=\operatorname{Gal}\left(K_{\infty} / K_{\text {cyc }}\right)$ and $\Gamma=\operatorname{Gal}\left(K_{\text {cyc }} / K\right)$. Then $G \cong \mathbb{Z}_{p} \rtimes \mathbb{Z}_{p}$ is a pro- $p$, non-commutative $p$-adic Lie group of dimension 2 and is without $p$-torsion. The groups $H$ and $\Gamma$ are both isomorphic to $\mathbb{Z}_{p}$. The finite set $S$ of primes of $K$ consists of the infinite primes and the primes lying over $m N p$.

REMARK 11. Let $\left(K_{\infty} / K\right)$ be the false Tate curve extension described above. Then we can simplify the proof of Theorem 10, as explained below.

In this case $H \cong \mathbb{Z}_{p}$. So we have $\mathbb{H}_{\mathcal{F}}^{\text {ord }}[[H]] \cong(O[[W]])[[H]] \cong\left(O\left[\left[T_{1}\right]\right]\right)\left[\left[T_{2}\right]\right]$ for some finite extension $O$ of $\mathbb{Z}_{p}$ (by (Nor)). Also for any $p_{\xi}$, a height 1 prime in $O[[W]], O[[W]] / p_{\xi} \cong O_{\xi}$ holds. Hence we apply the structure theorem of finitely generated modules over $\mathbb{H}_{\mathcal{F}}^{\text {ord }}[[\mathcal{H}]] \cong O\left[\left[T_{1}, T_{2}\right]\right]$ (see $[\mathrm{N}-\mathrm{S}-\mathrm{W}]$ ), which provides us with a characteristic element $h \in O\left[\left[T_{1}, T_{2}\right]\right]$ for $\mathcal{Y}\left(\mathcal{T} / K_{\infty}\right)$. For any $p_{\xi}$ not dividing this $h$, we get by the same structure theorem that the $O\left[\left[T_{1}, T_{2}\right]\right]$ rank of $\mathcal{Y}\left(\mathcal{T} / K_{\infty}\right)$ is same as the $O_{\xi}\left[\left[T_{2}\right]\right]$ rank of $\mathcal{Y}\left(\mathcal{T} / K_{\infty}\right) / p_{\xi}$. Also in this case, by $\left(\mathbf{D i m}_{S}\right)$ for each $v$ in $S, \operatorname{dim} G_{v}=\operatorname{dim} G=2$. Hence there are only finitely many primes in $K_{\infty}$ lying over each prime $v$ in $S$. Then $K_{v}^{0}\left(A_{f_{\xi}} / K_{\infty}\right)$ is itself a cofinitely generated $O_{f}$ module for every $\xi$. Using this in the proof of Theorem 4, it is clear that both the kernel and the cokernel of $r_{\xi}$ are cofinitely generated $O_{f}$ module. Thus by applying the same theorem for every $\xi$, we get that both $\mathcal{Y}\left(\mathcal{T} / K_{\infty}\right) / p_{\xi}$ and $Y\left(\mathcal{T}_{\xi} / K_{\infty}\right)$ have the same $O_{\xi}\left[\left[T_{2}\right]\right]$ rank. Finally it is clear that the $O\left[\left[T_{1}, T_{2}\right]\right]$ rank of $\mathcal{Y}\left(\mathcal{T} / K_{\infty}\right)$ is a lower bound of the $O\left[\left[T_{1}, T_{2}\right]\right] / p_{\xi}$ rank of $\mathcal{Y}\left(\mathcal{T} / K_{\infty}\right) / p_{\xi}$ for any $\xi \in \mathfrak{X}_{\text {arith }}\left(\mathbb{H}_{\mathcal{F}}^{\text {ord }}\right)$. From these discussions the theorem follows.

Numerical Example. Let $E$ be the elliptic curve $79 A 1$ of conductor 79 in Cremona's Table. Take $p=3$. Consider the false Tate curve extension $K_{\infty} / K$ with $K=\mathbb{Q}\left(\mu_{3}\right)$ and $K_{\infty}=\mathbb{Q}\left(\mu_{3^{\infty}}, \bigcup_{n \geq 1} m^{1 / 3^{n}}\right)$, where $m=79^{n}$ for any $n$ such that 3 does not divide $n$. Now $v_{3}$, the unique prime over 3 in $K$, is a prime of good, ordinary reduction for $E$ in $K$ and $E$ has non-split multiplicative reduction at both the primes over 79 in $K$. By [D-D, Theorem A.37], we get that the dual Selmer group $X\left(E / K_{\text {cyc }}\right)$ is isomorphic to $\mathbb{Z}_{3}$ and also we can deduce that the dual Selmer group $X\left(E / K_{\infty}\right)$ has $\Lambda(H)$ rank 1 . Now $(0,0)$ is a point of infinite order of $E(K)$. Applying [C-S, Proposition 4.9], the $\Lambda(H)$ rank of the dual fine Selmer group $Y\left(E / K_{\infty}\right)$ is strictly less than that of the dual Selmer group $X\left(E / K_{\infty}\right)$. Since the later rank is 1 , the former rank is zero. Hence $Y\left(E / K_{\infty}\right)$ is pseudonull as a $\Lambda_{\mathbb{Z}_{p}}(G)$ module i.e. conjecture B holds for $E$. In this case we can take $S=\{3,79\}$. Now by $[\mathrm{H}-\mathrm{V}]$ the hypothesis $\left(\mathbf{D i m}_{S}\right)$ is satisfied. Further, the residual representation is irreducible for $p=3$. Hence if we take the Hida family associated to $f_{E}$, the weight 2 newform in $S_{2}\left(\Gamma_{0}(79)\right)$ corresponding to $E$, then we see that in a branch of the Hida family associated to $f_{E}$, for all but finitely many members $g$, Conjecture B holds for $Y\left(T_{g} / K_{\infty}\right)$. 
4. Selmer group. We continue with the notation used in Section 2. All the results in this section for the Selmer groups can be proved in a way entirely analogous to the corresponding assertions for the fine Selmer groups, and hence we skip all the proofs.

For a finite extension $L$ of $K$ and each $v$ not lying over $p$ in $S$, we define

$$
J_{v}^{i}\left(A_{f} / L\right):=\underset{w \mid v}{\oplus} H^{i}\left(L_{w}, A_{f}\right)
$$

and for $v_{p}$, a prime lying over $p$,

$$
J_{v_{p}}^{i}\left(A_{f} / L\right):=\underset{w \mid v_{p}}{\oplus} H^{i}\left(I_{w}, A_{f} / A_{f}^{+}\right)
$$

for $i=0,1,2$, where for each prime $w$ in $L$ lying over $v, L_{w}$ denotes a completion of $L$ at $w$, and $I_{w}$ is the corresponding inertial subgroup and $A_{f}^{+}$, as usual, is defined using the filtration of $A_{f}$ as a $\mathrm{G}_{\mathbb{Q}_{p}}$-module which is obtained using the ordinarity of $f$ at $p$. For an infinite extension $L_{\infty}$ of $K$, the definition of $J_{v}^{i}\left(A_{f} / L_{\infty}\right)$ extends by taking the inductive limit of $J_{v}^{i}\left(A_{f} / L^{\prime}\right)$ over all finite extension $L^{\prime}$ of $K$ contained in $L_{\infty}$. We now define the Greenberg Selmer group or simply the Selmer group of $f$ over $\mathcal{L} / K$ for the lattice $T_{f}$, denoted by $S\left(A_{f} / \mathcal{L}\right)$, as

$$
\text { Definition 2. } S\left(A_{f} / \mathcal{L}\right)=\operatorname{Ker}\left(H^{1}\left(K_{S} / \mathcal{L}, A_{f}\right) \longrightarrow \underset{v \in S}{\oplus} J_{v}^{1}\left(A_{f} / \mathcal{L}\right)\right) \text {. }
$$

The Pontryagin dual of $S\left(A_{f} / \mathcal{L}\right)$, denoted by $X\left(T_{f} / \mathcal{L}\right)$, is a finitely generated (left) module over the Iwasawa algebra $O_{f}[[\mathcal{G}]]$. As $K / \mathbb{Q}$ is abelian, it is a deep result of Kato (see $[\mathrm{Ka}]$ ) that $X\left(T_{f} / K_{\text {cyc }}\right)$ is a finitely generated torsion $O_{f}[[\Gamma]]$ module. We state the following hypothesis.

$(\mu=0)$ : The dual Selmer group $X\left(T_{f} / K_{\text {cyc }}\right)$ is a finitely generated $O_{f}$ module.

We remark that unlike the case of the fine Selmer group $Y\left(T_{f} / K_{\text {cyc }}\right)$, there are examples known where $X\left(T_{f} / K_{\text {cyc }}\right)$ is not finitely generated over $\mathbb{Z}_{p}$. However under the assumption (Irr) and for $K=\mathbb{Q}$, a conjecture of Greenberg [Gr, Conjecture 1.11 asserts that for an elliptic curve $E,(\mu=0)$ holds for $X\left(T_{p} E / \mathbb{Q}_{\text {cyc }}\right)$.

Assume that $(\mu=0)$ holds for $X\left(T_{f} / K_{\text {cyc }}\right)$. Then it is easy to see (similar to Lemma 2) that the kernel and the cokernel of the map

$$
X\left(T_{f} / \mathcal{L}\right)_{\mathcal{H}} \longrightarrow X\left(T_{f} / K_{\text {cyc }}\right)
$$

are finitely generated $O_{f}$-modules. Thus using Nakayama's Lemma and assuming $(\mu=0)$ hypothesis, we see that $X\left(T_{f} / \mathcal{L}\right)$ is a finitely generated $\Lambda_{O_{f}}(\mathcal{H})$ module.

Next we define the Selmer group associated to the large Galois representation (2). Let $S$ be chosen as before. The definition of the Selmer group of the $\Lambda$-adic representation over the admissible $p$-adic Lie extension $\mathcal{L} / K$, denoted by $\mathcal{S}(\mathcal{A} / \mathcal{L})$, is obtained by replacing $A_{f}$ with $\mathcal{A}$ in the Definition 2. The Pontryagin dual of $\mathcal{S}(\mathcal{A} / \mathcal{L})$, denoted by $\mathcal{X}(\mathcal{T} / \mathcal{L})$ can be given the structure of an $\mathbb{H}_{\mathcal{F}}^{\text {ord }}[[\mathcal{G}]]$-module in a natural way. It can be shown (see [Oc, Proposition 4.9$]$ ) that $\mathcal{X}\left(\mathcal{T} / K_{\text {cyc }}\right.$ ) is a finitely generated $\mathbb{H}_{\mathcal{F}}^{\text {ord }}[[\Gamma]]$ module. The following theorem is essentially due to $[\mathrm{E}-\mathrm{P}-\mathrm{W}]$ and [Oc]. It can also be proved along the lines of [J-S, Theorem 8].

THEOREM 12. The following are equivalent. 
1. There exists $\xi_{0} \in \mathfrak{X}_{\text {arith }}\left(\mathbb{H}_{\mathcal{F}}^{\text {ord }}\right)$ such that $(\mu=0)$ is true for $X\left(T_{f_{\xi_{0}}} / K_{\text {cyc }}\right)$.

2. For every $\xi \in \mathfrak{X}_{\text {arith }}\left(\mathbb{H}_{\mathcal{F}}^{\text {ord }}\right),(\mu=0)$ is true for $X\left(T_{f_{\xi}} / K_{\text {cyc }}\right)$.

3. $\mathcal{X}\left(\mathcal{T} / K_{\text {cyc }}\right)$ is a finitely generated $\mathbb{H}_{\mathcal{F}}^{\text {ord }}$-module.

We assume one of the equivalent conditions of Theorem 12 holds. Again, it is easy to see that the kernel and the cokernel of the map

$$
\mathcal{X}(\mathcal{T} / \mathcal{L})_{\mathcal{H}} \longrightarrow \mathcal{X}\left(\mathcal{T} / F_{\text {cyc }}\right)
$$

are finitely generated $\mathbb{H}_{\mathcal{F}}^{\text {ord }}$-modules and hence using Nakayama's Lemma we see that $\mathcal{X}(\mathcal{T} / \mathcal{L})$ is a finitely generated $\mathbb{H}_{\mathcal{F}}^{\text {ord }}[[\mathcal{H}]]$ module. Thus in this case, we know that both $\mathcal{X}(\mathcal{T} / \mathcal{L}) / p_{\xi}$ and $\mathcal{X}\left(T_{\xi} / \mathcal{L}\right)$ are finitely generated modules over $O_{\xi}[[\mathcal{H}]]$ for any arithmetic point $\xi$.

Recall that for an arithmetic point $\xi$, we have $\mathcal{A}\left[p_{\xi}\right] \cong A_{f_{\xi}}$, the discrete lattice associated to the eigenform $f_{\xi}$. The control theorem in this case is as follows.

TheOREm 13. Assume that the conditions (Nor) and (Irr) hold. Then the Pontryagin dual of the kernel and the cokernel of the map

$$
S\left(\mathcal{A}\left[p_{\xi}\right] / \mathcal{L}\right) \stackrel{s_{\xi}}{\longrightarrow} \mathcal{S}(\mathcal{A} / \mathcal{L})\left[p_{\xi}\right]
$$

are finitely generated $O_{\xi}[[\mathcal{H}]]$ modules for all $\xi \in \mathfrak{X}_{\text {arith }}\left(\mathbb{H}_{\mathcal{F}}^{\text {ord }}\right)$ and are in fact $O_{\xi}[[\mathcal{H}]]$ torsion i.e. $O_{\xi}[[\mathcal{G}]]$ pseudonull for all but finitely many such $\xi$.

REMARK 14. Note that we do not need to assume that one of the equivalent conditions of Theorem 12 for proving this control theorem.

Assume that one of the equivalent conditions of Theorem 12 holds. Let dimension of $\mathcal{G}$ is $\geq 2$. Then both $\mathcal{X}(\mathcal{T} / \mathcal{L}) / p_{\xi}$ and $X\left(T_{\xi} / \mathcal{L}\right)$ are finitely generated $O_{\xi}[[\mathcal{H}]]$ modules and it is immediate from the above Theorem 13 that for all but finitely many $\xi \in \mathfrak{X}_{\text {arith }}\left(\mathbb{H}_{\mathcal{F}}^{\text {ord }}\right)$, the $O_{\xi}[[\mathcal{H}]]$ rank of $\mathcal{X}(\mathcal{T} / \mathcal{L}) / p_{\xi}$ and $X\left(T_{\xi} / \mathcal{L}\right)$ are the same. Also by Lemma 5 , for all but finitely many $\xi$, the $\mathbb{H}_{\mathcal{F}}^{\text {ord }}[[\mathcal{H}]]$ rank of $\mathcal{X}(\mathcal{T} / \mathcal{L})$ and the $O_{\xi}[[\mathcal{H}]]$ rank of $\mathcal{X}(\mathcal{T} / \mathcal{L}) / p_{\xi}$ are the same. Combining this with Theorem 13 , we get the following result.

Proposition 15. Assume (Nor), (Irr), and $K / \mathbb{Q}$ is abelian. Also assume that $(\mu=0)$ holds for some $\xi_{0} \in \mathfrak{X}_{\text {arith }}\left(\mathbb{H}_{\mathcal{F}}^{\text {ord }}\right)$. Then for all but finitely many $\xi \in$ $\mathfrak{X}_{\text {arith }}\left(\mathbb{H}_{\mathcal{F}}^{\text {ord }}\right)$, the $O_{\xi}[[\mathcal{H}]]$ rank of $X\left(T_{\xi} / \mathcal{L}\right)$ are the same with the rank being equal to the $\mathbb{H}_{\mathcal{F}}^{\text {ord }}[[\mathcal{H}]]$-rank of $\mathcal{X}(\mathcal{T} / \mathcal{L})$.

REMARK 16. Let $K=\mathbb{Q}\left(\mu_{p}\right), \mathcal{L}=K_{\infty}$ correspond to the false Tate curve extension. In this case [Sh] has shown that under the hypothesis of Proposition 15, the $O_{\xi}[[\mathcal{H}]]$ ranks of $X\left(T_{\xi} / \mathcal{L}\right)$ are the same for all $\xi \in \mathfrak{X}_{\text {arith }}\left(\mathbb{H}_{\mathcal{F}}^{\text {ord }}\right)$. He has also given a numerical example where the above proposition holds for the Hida family associated to $X_{0}(11)$. However his methods do not seem to generalize easily to a general admissible $p$-adic Lie extension.

Acknowledgements. We would like to thank R. Sujatha for many helpful discussions and comments. We thank TIFRCAM, Bangalore for the hospitality accorded in the final stage of preparation of this article. The author gratefully acknowledges the support from the SPM scholarship of CSIR, India. We also thank the referee for his comments. 


\section{REFERENCES}

[Ca] H. CARAYOL, Formes modulaires et représentations galoisiennes à valeurs dans un anneau local complet, $p$-adic monodromy and the Birch and Swinnerton-Dyer conjecture (Boston, MA, 1991), Contemp. Math., 165, Amer. Math. Soc. (1994), pp. 213-237.

[D-D] T. DockChitser AND V. DockChistser, Computations in non-commutative Iwasawa Theory, with an appendix by J. Coates and R. Sujatha, Proc. London Math. Soc. (3), 94 (2007), pp. 211-272.

[C-S] J. Coates And R. Sujatha, Fine Selmer group of elliptic curves over p-adic Lie extensions, Math. Ann., 331:4 (2005), pp. 809-839.

[E-P-W] M. Emerton, R. Pollack and T. Weston, Variation of Iwasawa invariants in Hida families, Invent. Math., 163:3 (2006), pp. 523-580.

[Gr] R. Greenberg, Iwasawa Theory of Elliptic Curves, in Arithmetic Theory of Elliptic Curves, Lecture Notes in Mathematics, 1716 (1999), pp. 51-144.

[Ho1] S. Howson, Euler characteristic as invariants of Iwasawa modules, Proc. London Math. Soc. (3), 85 (2002), pp. 634-658.

[Ho2] S. Howson, Structure of central torsion Iwasawa modules, Bull. Soc. Math. France, 130:4 (2002), pp. 507-535.

[Hi] H. HIDA, Galois representations into $\mathrm{GL}_{2}\left(\mathbb{Z}_{p}[[X]]\right)$ attached to ordinary cusp forms, Invent. Math., 85:3 (1986), pp. 545-613.

[J-S] S. Jha AND R. Sujatha, On the Hida deformation of fine Selmer group, Journal of Algebra, 338:1 (2011), pp. 180-196.

[Ka] K. KATO, p-adic Hodge theory and values of zeta functions of modular forms, Cohomologies $p$-adiques et applications arithmétiques. III. Astérisque, 295:ix (2004), pp. 117290.

[M-W] B. Mazur AND A. Wiles, On p-adic analytic families of Galois representations, Compositio. Math., 59 (1986), pp. 231-264.

[N-S-W] J. Neukirch, A. Schmidt And K. Winberg, Cohomology of Number Fields, Grundl. math. Wiss., 323, Springer, 2000.

[Oc] T. OchiAI, On the two-variable Iwasawa main Conjecture, Compositio. Math., 142 (2006), pp. $1157-1200$

[Sh] A. C. Sharma, Iwasawa invariants for the False Tate extension and congruences between modular forms, J. Numb. Th., 129 (2009), pp. 1893-1911.

[Ve] O. Venjakob, A non-commutative Weierstrass preparation theorem and applications to Iwasawa theory, Crelle J., 559 (2003), pp. 153-191.

[Wi] A. Wiles, On ordinary $\lambda$-adic representations associated to modular forms, Invent. Math., 94:3 (1988), pp. 529-573. 
